\title{
Breaking an unhelpful circle, where innovation and regularia delay clinical practice for patient benefit
}

\author{
Peter Ell ${ }^{1,2} \cdot$ Ignasi Carrio ${ }^{3,4} \cdot$ Arturo Chiti ${ }^{5,6}$ \\ Published online: 3 April 2019 \\ (C) Springer-Verlag GmbH Germany, part of Springer Nature 2019
}

Not that long ago, some 10 to 15 years ago, it seemed relatively simple to translate bench to practice, innovation to patient benefit. Whilst regulations were in place, they were not so onerous as to impede the acceptance of novel tools and methodologies for patient care. Some of many examples include the development and application of myocardial perfusion imaging, the investigation of postsynaptic status with specific probes permitting the early detection of parkinsonian syndromes, and the study of cerebral blood flow patterns in patients with dementias. This seemed a golden age. Innovation was stimulated, scientific discoveries made and published, novel clinical practice emerged, and patients benefited. Interestingly, costs seemed containable, developments were rapidly diffused, and all stakeholders, providers and purchasers of health care and industrial/commercial as well as investigator partners seemed, all in all, to be "in business".

Alas, in recent years the above scenario has changed dramatically. Health-care providers are adamant that health costs need to be not just contained but also curtailed. The public has begun to be on the receiving end of cost considerations and delays in the provision of care. No public consultation has ever taken place on the appropriateness of such policies,

\section{Arturo Chiti}

arturo.chiti@hunimed.eu

1 University College London, London, UK

2 University College Hospitals, London, UK

3 Autonomous University of Barcelona, Barcelona, Spain

4 Hospital Sant Pau, Barcelona, Spain

5 Department of Biomedical Sciences, Humanitas University, Rozzano, Italy

6 Unit of Nuclear Medicine, Humanitas Research Hospital, Rozzano, Italy whilst the professions have been given the mantra that care should be purchased on the basis of evidence, not withstanding the fact that most of the evidence for evidence-based care was obtained in the past. Progress cannot wait for standard evidence gathering, but the requirement that care should be evidence-based provides an almost perfect delaying mechanism that controls innovation and its diffusion into clinical practice.

There are constraints in addition to those discussed above preventing the rapid accrual of data that will ultimately provide the evidence required by the authorities and acceptance by those who purchase and provide health care. What are these?

"Publish or perish" is a major source of concern [1]. The inappropriate weight given to impact factors in the promotion of academic staff has led to the inflated need to publish rapidly and be "first". This in turn has led to the emergence of a plethora of publications which, whilst meritorious, do not have the statistical power and fail the requirements needed for evidence based data analysis. At present, multiple new exciting probes have emerged in our field, and promise a new and exciting era for nuclear medicine. But the problems described above have led to delay in the approval of these novel technologies by purchasing authorities and hence their diffusion into the clinic to benefit patients.

In many circumstances, after the original reports on new probes that have emerged or are soon to emerge (e.g. assessment of amyloid deposition, investigation of the presence or absence of tau, the introduction of PSMA probes for diagnosis and treatment, and several others), 5 or more years may elapse without formal trial data clearly showing their benefit in the context of disease management and patient care. Hence, purchasing authorities delay necessary authorizations, and often blame practitioners for not having provided sufficiently robust data to allow approval and reimbursement.

The second issue, also related to the above, is the restricted concept of the principal investigator (PI) as the sole 
beneficiary of a grant or academic recognition. When a physician identifies a clinical problem requiring a solution, the biologist identifies the target, the radiochemist synthesizes the specific tracer and the physicist develops the algorithms required to deal with low-signal image processing, who deserves recognition as the PI? At a time when team work is not only essential but also widely recognized as the most important factor in the achievement of success, the PI concept needs major overhaul.

The above two issues illustrate why it has been so hard to develop an infrastructure that can deliver rapid multicentre, transnational, cost-sparing, clinical trials. At times, even relevant industry partners have failed to appreciate the above, and have dispersed multiple smaller trials to many centres, all eager to publish and be first.

If we want nuclear medicine to benefit from the rapid progress being made in the development of novel radiolabelled probes (the basis of what makes our field distinct from all other imaging modalities), the above issues need to be addressed with significant solutions as soon as they are recog- nized. Perhaps a consensus-building conference needs to be called by the European and other International Organisations, so that a strategy can be agreed upon to deal with the issues outlined. This will not be easy. Many particular interests are involved and need open discussion. But the goal is worthwhile, if we keep in mind our main objective, namely the timely improvement in the care delivered to our patients.

\section{Compliance with ethical standards}

This article does not describe any studies involving humans or animals.

Conflicts of interest None.

\section{Reference}

1. Finkel A. To move research from quantity to quality, go beyond good intentions. Nature. 2019;566:297.

Publisher's note Springer Nature remains neutral with regard to jurisdictional claims in published maps and institutional affiliations. 